\title{
The Spread of Coronavirus in The Air
}

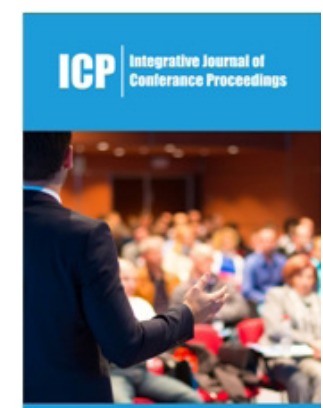

*Corresponding author: Solomon I Khmelnik, Department of Fuel-Free Energy, Israel

\section{Submission: August 14, 2020}

Published:

Volume 2 - Issue 3

How to cite this article: Solomon I Khmelnik. The Spread of Coronavirus in The Air. Int J Conf Proc.2(3). ICP.000536.2020.

Copyright@ Solomon I Khmelnik, This article is distributed under the terms of the Creative Commons Attribution 4.0 International License, which permits unrestricted use and redistribution provided that the original author and source are credited.

\author{
Solomon I Khmelnik ${ }^{1 *}$ \\ ${ }^{1}$ Department of Fuel-Free Energy, Israel
}

\section{Annotation}

The question of how the coronavirus spreads in the air is being considered. It is shown that the existing ideas about the spread of the virus as particles are unconvincing. It is shown that the coronavirus can spread as an electromagnetic wave modulated by the natural frequency of the coronavirus. Numerous analogies are pointed out. Possible methods of dealing with the pandemic are proposed, which require studying the physics of the propagation of high-frequency electromagnetic waves in the air and the interaction of organic molecules through the exchange of electromagnetic radiation.

\section{Introduction}

The person is infected with the coronavirus but does not yet know about it. A few days later, it turns out that all those who were with him in the same large room are infected with the coronavirus. How many viruses did this person shed (at a very early stage) and how quickly did they spread in the air? The document (which was signed by 239 scientists from 32 states) claims that the virus can be in the air in the form of smaller particles than previously thought, and this allows COVID-19 molecules to enter the body when breathing. Also approved that the virus quickly settles on the surface because it is contained in large heavy droplets (note that these two statements contradict each other). According to Benedetta Allegranzi, WHO's the technical director for infection control, the evidence given by scientists is inconclusive. She noted that there is still debate on this topic in scientific circles [1]. Let's join this debate.

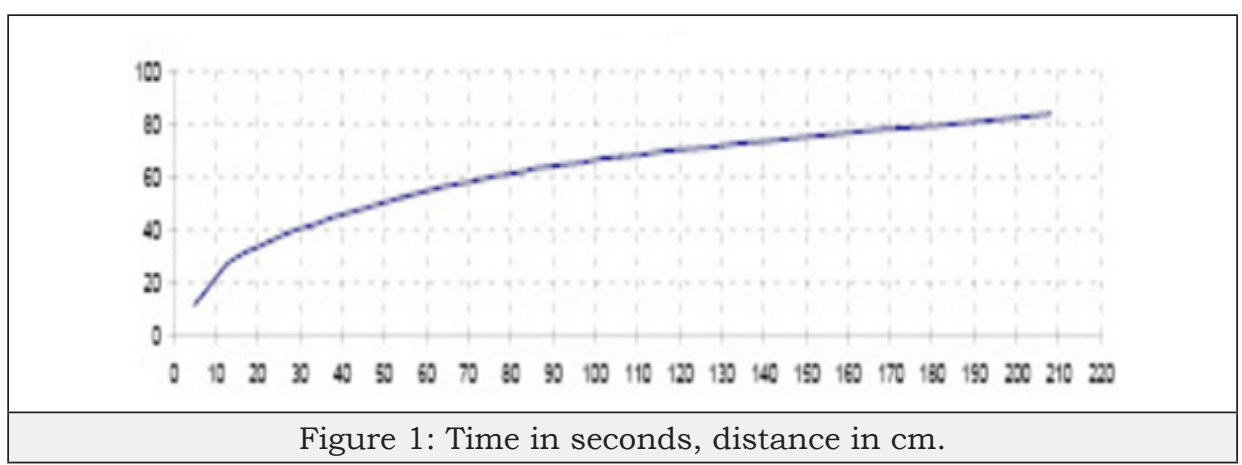

Let us assume that the particles of the virus are commensurate with the molecules of odorous substances and are excreted by a sick person as intensely as the molecules of these substances. It is known that molecules of a substance move by molecules of air, and for this there are two possible ways: diffusion (the movement of molecules of a substance, which is created by the thermal movement of air molecules) and convection (the movement of molecules of a substance, which is created by the uneven distribution of air molecules, not substance). A comparison of these methods (including through experimentation) in the spread of odor in the air is considered in [2,3]. It shows, for example, that due to diffusion, alcohol vapors in the air spread over a distance of $1 \mathrm{~m}$ in 14 hours. The spread by convection (when creating an increased density of ammonia) of the smell of smoke over a distance of $0.8 \mathrm{~m}$ occurs in 2 minutes. In Figure 1 shows the experimentally obtained dependence of the spread 
of the smoke boundary on time for a $3 \mathrm{~m}$ long pipe (where there is an increased density of ammonia) at a low concentration of smoke (but our infecting person has also not yet managed to gain a large concentration of viruses).

It can be seen that after the smoke spreads to a distance of up to $2 \mathrm{~m}$, it practically stops moving. The authors conclude that convection plays the main role in the mechanism of odor propagation. We agree with them because we don't argue with experiments. The only one question remains: why is it necessary to assume that the source of the odor is in the area of increased air concentration? If this increased concentration of air is absent, then no reason for the spread of the smell is visible at all (except, of course, the increased kinetic energy of the molecules of the substance). The same can be said about the reasons for the spread of the coronavirus. How, then, does the smell spread? The question, of course, is not that, but, one must think, the answer will be the same for our case. If difficulties arise with the spread of the smell, then how do ants survive - after all, it is argued that it is by a smell that they find food and communicate with the help of pheromones, releasing, for example, an alarm pheromone when injured or killed. The assumption arises that it is not necessary to transfer a substance to transmit information. 0 ! This is an old idea of homeopaths and (I think) there will be 239 more researchers from 32 countries who will come up with a refutation. But I believe in the objectivity of scientists and therefore I will continue.

\section{The electromagnetic volume standing wave}

It was shown in $[4,5]$ (as a consequence of solving Maxwell's equations) that an electromagnetic standing wave can exist in a limited volume of vacuum. This volume may have a variety of shapes. A standing wave is not emitted through the faces of the shapes and, in the absence of external energy flows, such a wave retains its energy, frequency and volume shape. This electromagnetic wave can be modulated at a lower frequency. In this case, this volume turns into a keeper of energy and information. In [5-7], well-known experiments and natural phenomena are considered, which serve as proof of the existence of such a keeper.

A water molecule is a volume that stores a standing wave. In this case, the standing wave is stored in the space between the oxygen and hydrogen atoms, i.e. in a vacuum-see Figure 2. Molecules of nitrogen and oxygen in the air are also the volume that stores a standing wave. In this case, the standing wave is stored in the space between the atoms of these molecules, i.e. in a vacuum. In [7] it is shown that on the basis of these facts the peculiarities of homeopathy can be explained, incl. enhancement of the therapeutic effect during dilution and the absence of the influence of impurities. In [8], on the basis of the same facts, it is shown that a highly organized structure can exist in the air, comparable in intelligence to the brain of an animal. Such a structure can be the collective brain of a community of bio-organisms.

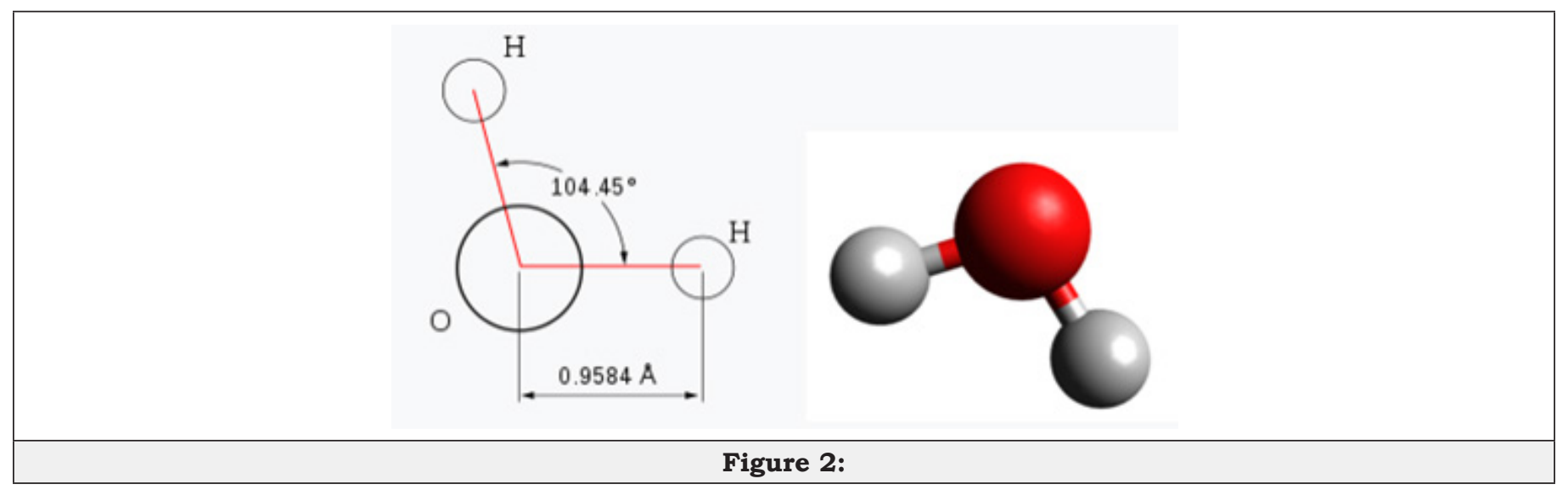

So, it is possible to indicate stable volumes, where a bulk standing electromagnetic wave can be located. These options are listed in Table 1. Table 1 also shows molecules in organics. The mutual arrangement of these molecules remains unchanged. In this case, a free vacuum space with linear dimensions of about $3\left[\cdot 10 \rrbracket^{\wedge}(-9)\right.$ $\mathrm{m}$ remains between the molecules. A standing wave also appears in this stable volume. It can be assumed that there is a standing wave of a certain frequency in the coronavirus-the natural freTable 1: quency of the coronavirus. It can be assumed that this frequency is comparable to the natural frequency of drug molecules [9], which is within $\mathrm{L}=\left(10^{\wedge} 13 \llbracket \div 10 \rrbracket^{\wedge} 14\right) \mathrm{Hz} \mathrm{Hz}$, which is much lower than the frequencies listed in Table. 1 . These frequencies can modulate the frequency of the electromagnetic field in air molecules. Note that the region of existence of a standing wave with such a frequency is approximately $\left[10 \rrbracket^{\wedge} 7\right.$ times larger than the region of a standing wave in an air molecule.

\begin{tabular}{|l|c|c|c|c|}
\hline & Water & Nitrogen & Oxygen & Organics \\
& $\mathbf{H}_{2} \mathbf{0}$ & $\mathbf{N}_{2}$ & $3.10^{-9}$ & $3.10^{-9}$ \\
\hline
\end{tabular}




\begin{tabular}{|c|c|c|c|c|}
\hline Molecule size (m) & $3.10^{-10}$ & $3.10^{-10}$ & $3.10^{-10}$ & $10^{-8}$ \\
\hline Location of the standing wave region & $\begin{array}{l}\text { Between oxygen and } \\
\text { hydrogen atoms }\end{array}$ & $\begin{array}{l}\text { Between nitrogen } \\
\text { atoms }\end{array}$ & $\begin{array}{c}\text { Between oxygen } \\
\text { atoms }\end{array}$ & $\begin{array}{l}\text { Between molecules } \\
\text { in organics }\end{array}$ \\
\hline Atom radius $\left(\mathrm{pm}=10^{-12} \mathrm{~m}\right)$ & $53 \mathrm{pm}$ & $56 \mathrm{pm}$ & $48 \mathrm{pm}$ & \\
\hline Interatomic distance $\left(A=10^{-10} \mathrm{~m}\right)$ & $0.9584 \mathrm{~A}$ & $1.095 \mathrm{~A}$ & $1.2074 \mathrm{~A}$ & \\
\hline $\mathrm{R}(\mathrm{m})$ & $5.10^{-11}$ & $5.10^{-11}$ & $6.10^{-11}$ & $1.5 .10^{-9}$ \\
\hline$\omega\left(\mathrm{s}^{-1}\right)$ & $10^{19}$ & $10^{19}$ & $1.2 .10^{19}$ & $5.10^{16}$ \\
\hline $\mathrm{f}(\mathrm{Hz})$ & $1.5 .10^{18}$ & $1.5 .10^{18}$ & $1.8 .10^{18}$ & $10^{16}$ \\
\hline$\gamma(\mathrm{m})$ & $2.1^{-10}$ & $2.10^{-10}$ & $2.4 .10^{-10}$ & $3.10^{-8}$ \\
\hline
\end{tabular}

For brevity, we will call a standing wave of air an $\omega \_v$-wave, and a standing wave of a coronavirus an $\omega_{-} 0$-wave. Since $\omega_{-} 0 \ll \omega_{-} v$, then $\omega_{-} 0$-wave of the coronavirus can modulate the $\omega_{-} v$-wave of an air molecule, and this modulation can be transmitted between air molecules. Thus, the transmission of a modulated wave can occur without the directed motion of molecules - their collision is enough. We will consider the effect of modulated air molecules on the body below. But for now, let us note that one molecule is enough for "contaminated air molecules" to appear in a large volume of air. Moreover, modulation of air molecules by different specimens of the same coronavirus creates a phase difference of electromagnetic oscillations in different air molecules. The exposure depends on the cumulative exposure of all "contaminated air molecules". It is obvious that the total impact of molecules with different phases is less than the total impact of "in-phase molecules". Similarly, in [7] it is explained that dilution of the medicinal solution enhances the healing effect in homeopathy.

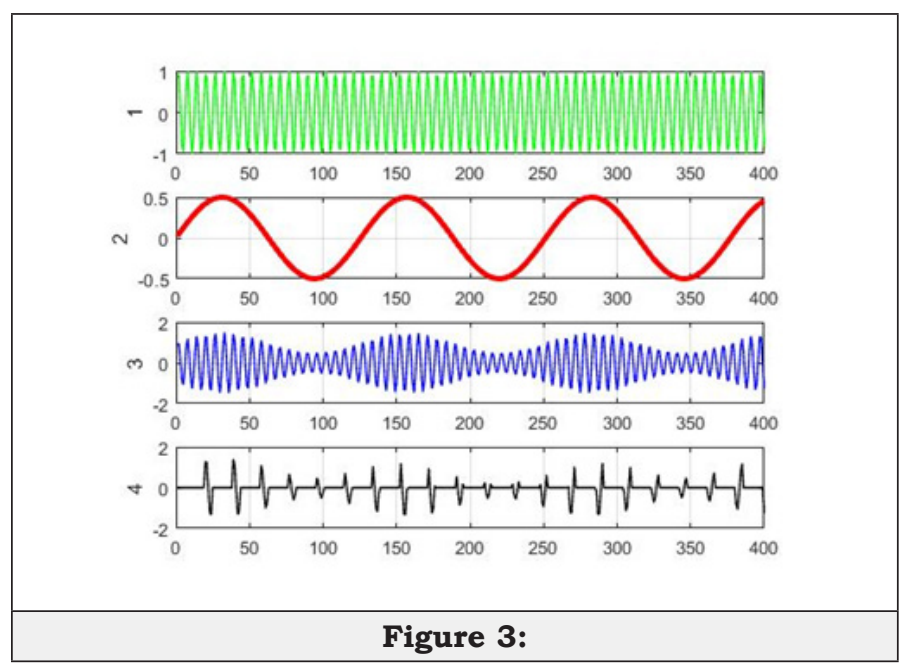

The foregoing is illustrated in Figure 3, where shown
A. $\omega_{-} v-w a v e$,
B. $\omega_{-} 0$-wave,

C. modulated wave $\omega_{-}$vo-wave obtained if standing $\omega_{-} 0-$ waves would cover space without gaps,

D. modulated wave is a $\omega_{-}$vo-wave obtained when standing $\omega_{-} 0$-waves are located in space with gaps.

\section{Infection with Coronavirus}

We first note the following. It is known that electromagnetic radiation interacts with molecules of a substance, causing radiation or absorption of electromagnetic radiation by molecules of a substance at certain frequencies [5]. Therefore, molecules of a substance can interact through electromagnetic radiation. A multiple enhancement of the effect of such an interaction appears when the natural frequencies of the emitting and absorbing molecules coincide. Meanwhile, numerous works are known, which show that all organs of animals and humans emit electromagnetic waves. These radiations are used in medical diagnostics. The effect of absorbed radiation on an organic molecule of matter is evidently in the enhancement and/or suppression of some functions of this molecule. Ultimately, this can be expressed in the transformation of the functional capabilities of the irradiated molecule, i.e. in her change. The coronavirus is known to transform the organic molecule it comes in contact with. For what follows, it is necessary to assume that it is the natural frequency of the coronavirus transforms this molecule. Then exposure to this frequency is sufficient without the presence of the coronavirus. In the same way, the natural frequency of a drug substance without the presence of the drug itself "cures a sick" molecule [7]. The only difference is that the natural frequency of the coronavirus "infects a healthy" molecule.

\section{Where is the exit?}

In the absence of the Internet, foreign influence was carried out through radio broadcasts. In the USSR, to combat "enemy voices" was used as the so-called jammers are noise generators operating at the frequency of the "enemy voice" transmission. For me, a former resident of the USSR, the first way to fight the coronavirus suggests itself: we need a jammer at the natural frequency of air molecules. Such a jammer can work in public places, but it can also be made in a form suitable for individual use. It is possible, however, that the natural frequency of air molecules is modulated by some frequencies useful for humans, for example, the natural frequencies of plants. Therefore, the frequency width of the white noise of the jammer should be limited to frequencies close to the natural frequency of the coronavirus. Another way could be as follows. In modern medicine (including non-traditional medicine), a method of searching for electromagnetic radiation with a frequency that cures 
a particular disease is widespread. Surely, in this case, frequencies with the opposite effect are detected. Therefore, it is necessary to look for a frequency that is fatal for the coronavirus. The proposed methods of dealing with the pandemic require, of course, the study of the physics of the propagation of high-frequency electromagnetic waves in the air and the interaction of organic molecules through the exchange of electromagnetic radiation.

\section{References}

1. (2020) Scientists have revised the way the coronavirus spreads in the air. Science and Technology, Russia.

2. Monastyrsky LM, Bondarev RV (2014) To the question about the mechanism of spreads of odor in the air. The Success of Modern Natural Science 12(Part 4): 448-450.
3. Monastyrsky LM, Bondarev RV (2015) To the question about the mechanism of spreads of odor in the air. The Success of Modern Natural Science 1(part 1): 132-134.

4. Khmelnik SI (2020) Inconsistency solution of Maxwell's equations. Mathematics in Computer Corp, Israel, pp. 1-374.

5. Khmelnik SI (2020) Electromagnetic keeper of energy and information. Canadian Journal of Pure and Applied Sciences 13(3): 4853-4859.

6. Khmelnik SI (2020) About the interaction of nanoparticles. Determinations in Nanomedicine \& Nanotechnology 1(4): 1-4.

7. Khmelnik SI (2020) To the rationale for homeopathy. Determinations in Nanomedicine \& Nanotechnology 2(1): 1-3.

8. Novakovskaya YV, Structure of molecules. Chemistry Faculty of Moscow State University, Russia.

9. Khmelnik SI (2020) Information transfer in biological systems by water and air. The Papers of Independent Authors 47: 51-61. 